\title{
Upgrades of the CMS muon detectors: from Run 3 towards HL-LHC
}

\section{Carlo Battilana* on behalf of the CMS Collaboration}

Università di Bologna ed Istituto Nazionale di Fisica Nucleare (INFN) - Sezione di Bologna, viale Berti Pichat 6/2 - 40127 - Bologna, Italy.

E-mail: carlo.battilana@cern.ch

The present CMS muon system consists of three different detector technologies: drift tubes (DT) and cathode strip chambers (CSC) are used in the barrel and endcap regions of the spectrometer as offline tracking and triggering devices, whereas resistive plate chambers (RPC) are installed both in barrel and endcaps and are exploited mostly in the trigger. To cope with the challenging conditions of increasing luminosity expected at HL-LHC, several upgrades of the muon detectors and trigger system are planned. In the case of DT and CSC, the electronics will be upgraded to handle higher rates, but there is no plan to replace the existing DT, CSC and RPC chambers. Therefore, accelerated ageing tests are being performed to assess the performance stability of all muon detectors under conditions which exceed, by one order of magnitude, the design specifications. New micropattern gas detectors will be added to improve the performance in the forward region, which is characterized by high background rates and a smaller, nonuniform magnetic field. Large-area triple-foil gas electron multiplier (GEM) detectors (GE1/1) are presently being installed during the second LHC long shutdown covering the pseudo-rapidity $(\eta)$ region $1.6<|\eta|<2.4$. They will limit the rate of background triggers, while preserving high trigger efficiency for low transverse momentum muons. For the HL-LHC operation, the muon forward region will also be enhanced with another large area GEM-based station (GE2/1) and with two new generation RPC stations, called RE3/1 and RE4/1, having low resistivity electrodes. These detectors will combine tracking and triggering capabilities and can stand particle rates up to few $\mathrm{kHz} / \mathrm{cm}^{2}$. In addition, an ME0 station of GEM chambers will be installed behind the new forward calorimeter to cover up to $|\eta|=2.8$ and take advantage of the pixel tracking coverage extension. We present results about the expected performance stability of the existing muon detectors at HL-LHC. Moreover, we report on the outcome of simulation-based studies, which describe the impact of the muon upgrades to the trigger and the reconstruction of muon physics objects.

European Physical Society Conference on High Energy Physics - EPS-HEP2019 - 10-17 July, 2019, Ghent, Belgium

\footnotetext{
* Speaker.
} 


\section{Introduction: the High-Luminosity LHC project}

During Run 2, which lasted from 2015 to 2018, the CERN Large Hadron Collider (LHC) provided proton-proton collisions at a centre of mass energy of $13 \mathrm{TeV}$, operating at instantaneous luminosity values up to $\sim 2.2 \cdot 10^{34} \mathrm{~cm}^{-2} \mathrm{~s}^{-1}$ and delivering a total integrated luminoisty around $160 \mathrm{fb}^{-1}$. After a shutdown period, Run 3 will occur from 2021 to 2023. Adiabatic improvements in instantaneous luminosity, and a potential centre of mass energy increase up to $14 \mathrm{TeV}$, are foreseen for Run 3. A major upgrade of the LHC and of its performance, the High Luminosity LHC (HL-LHC) [1] will start operations in 2026. HL-LHC is expected to deliver proton-proton collisions at $14 \mathrm{TeV}$ and reach, in its design (ultimate) upgrade scenario, instantaneous luminosities up to $\sim 5(7.5) \cdot 10^{34} \mathrm{~cm}^{-2} \mathrm{~s}^{-1}$, corresponding to an average pileup of 140 (200) collisions per bunch-crossing (BX), and collect a total integrated luminosity around $3000(4000) \mathrm{fb}^{-1}$.

At the HL-LHC, detectors such as the Compact Muon Solenoid (CMS) are expected to operate in conditions far beyond their design specifications. Most notably their components will be required to withstand particle fluxes and integrated doses that exceed the original specifications. Furthermore, to preserve present levels of acceptance for physics signatures at the electroweak scale, the triggers of HL-LHC experiments will need to be redesigned to operate at much higher rates.

\section{The CMS muon spectrometer: from present system to HL-LHC upgrade}

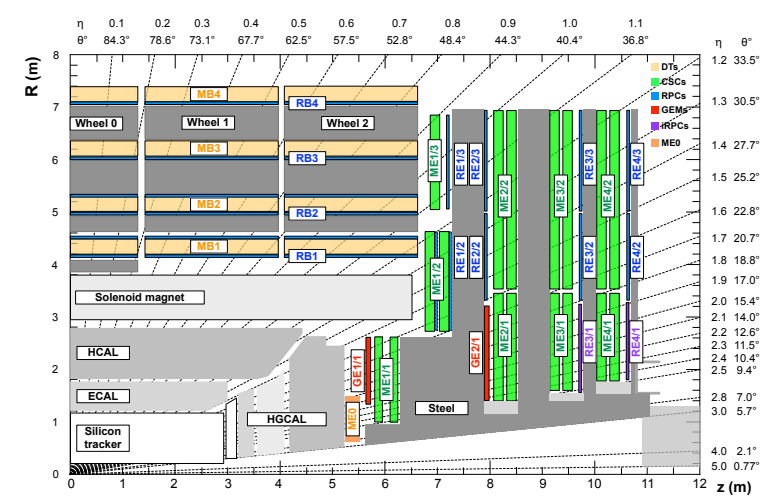

Figure 1: Longitudinal cross section of a quadrant of the CMS detector. The interaction point is located at the lower left corner of the figure.

The CMS muon system is designed for (i) an efficient offline identification of muons, (ii) a standalone triggering capability, and (iii) an improved measurement of the transverse momentum $\left(p_{\mathrm{T}}\right)$ of muons with energies greater than a few hundreds of $\mathrm{GeV}$. It presently consists of Drift Tubes (DT) and Cathode Strip Chambers (CSC), that serve as both tracking and triggering detectors, and cover the ranges from $|\eta|<1.2$ (DT) and $0.9<|\eta|<2.4$ (CSC). Resistive Plate Chambers (RPC) complement DT and CSC up to $|\eta|<1.9$, and are mostly used in the trigger.

The purpose of the muon system Phase-2 upgrade [2], planned for HL-LHC, is to preserve its existing performance in the harsh conditions described in the previous section. It involves a consolidation and upgrade of the electronics of the existing detectors, as well as the addition of 
new detector layers in the endcaps of the spectrometer, where background is larger and the CMS magnetic field is less intense and uniform. Chambers built of Gas Electron Multiplier (GEM) detectors will be installed in the ranges $1.6<|\eta|<2.4$ (GE1/1 and GE/2) and $2.1<|\eta|<2.8$ (ME0). The GE1/1 and GE2/1 will consist of two layers of triple-GEMs, whereas the ME0 are designed as six-layer chambers. The ME0 will extend the coverage of the existing muon system. Because of their good spatial resolution, GEMs will equip the endcap in regions close to the interaction point, to improve the standalone measure of the muon $p_{T}$. The two outermost layers of the spectrometer's endcaps will instead be equipped by improved RPCs (iRPC), which cover from $1.8<|\eta|<2.4$. Figure 1, shows a quadrant of the CMS muon system, highlighting the position of both the present detectors and the ones planned as part of the Phase-2 upgrade.

\begin{tabular}{|c||c|c|c|c|c|c|c|}
\hline & DT & CSC & RPC & iRPC & GE1/1 & GE2/1 & ME0 \\
& $0-1.2$ & $0.9-2.4$ & $0-1.9$ & $1.8-2.4$ & $1.6-2.15$ & $1.6-2.4$ & $2.0-2.8$ \\
\hline \hline neutron fluence $\left(10^{12} \mathrm{n} / \mathrm{cm}^{2}\right)$ & 0.4 & 40 & 1 & 7 & 20 & 12 & 200 \\
\hline total ionization dose $(\mathrm{kRad})$ & 0.12 & 10 & 2 & 3 & 3 & 7 & 490 \\
\hline hit rate $\left(\mathrm{Hz} / \mathrm{cm}^{2}\right)$ & 50 & 4500 & 200 & 700 & 1500 & 700 & 48000 \\
\hline \hline charge per wire $(\mathrm{mC} / \mathrm{cm})$ & 20 & 110 & - & - & - & - & - \\
\hline charge per area $\left(\mathrm{mC} / \mathrm{cm}^{2}\right)$ & - & - & 280 & 330 & 6 & 3 & 280 \\
\hline
\end{tabular}

Table 1: Intergral radiation dose, hit rate and accumulated charge for the muon detectors at the end of HL-LHC (design scenario: $\sim 5 \cdot 10^{34} \mathrm{~cm}^{-2} \mathrm{~s}^{-1}, 3000 \mathrm{fb}^{-1}$ ) [2].

The integral radiation doses, hit rates and accumulated charges that the most irradiated regions of the different detectors expect at the HL-LHC are reported in Tab. 1. Results are either derived from simulations, or computed from extrapolations of information recorded during Run 2. Based on these numbers, the longevity of the muon chambers is assessed by exploiting the Gamma Ray Irradiation Facility at CERN (GIF++), which provides a $14 \mathrm{TBq}{ }^{137} \mathrm{Cs}$ source emitting $662 \mathrm{keV}$ photons. At present, all existing detectors have been tested up to expected integrated doses and accumulated charges that exceed the ones expected at HL-LHC, showing either no or limited performance degradation due to ageing. Similarly, no significant effects are observed for the muon detectors proposed in the muon system upgrade. However, some of the electronic components equipping the existing system are not expected to withstand the integral radiation doses foreseen by the end of HL-LHC. In addition, the upgrade of the CMS Level-1 (L1) Trigger system will require the chamber electronics to operate at rates and latencies beyond the ones they are able to tolerate. For these reasons, electronics of all existing muon detectors will be upgraded. In the CSC, components will be replaced to prevent saturation effects at high trigger rate. For the DT, a complex ondetector electronic system will be replaced by TDC streaming hits to new offdetector electronics, which can exploit the ultimate chamber resolution in the trigger; presently, the ultimate resolution is not available for the trigger. Finally, the RPC electronics upgrade will improve the time resolution of readout and trigger from $25 \mathrm{~ns}$ to a few ns.

\section{CMS GEM detectors in the LHC Run 3}

GE1/1 chambers, are presently being installed in CMS and are expected to operate during Run 3 [3]. Their main physics motivation is the enhancment of muon trigger performance in the $1.6<|\eta|<2.1$ region. The GE1/1 measurement of two additional points along the muon trajectory, 
with a resolution a few hundreds of $\mu \mathrm{m}$, is expected to reduce significantly the $\mathrm{L} 1$ muon trigger rate in the endcap, as shown in Fig. 2 (left).

In 2017, a demonstrator consisting of $10 \mathrm{GE} 1 / 1$ chambers (slice test) was installed in CMS to gain expertise in installation and commissioning and to prepare for the full system upgrade. The installation of the chambers from the slice test allowed to highlight and overcome difficulties in the insertion of chambers int the CMS endcaps noses. Furthermore, it was possible to integrate GEMs in the CMS detector control system (DCS) and in the data acqusition system (DAQ). Finally, the slice test was successfully operated during CMS collision runs and the inclusion of GEM data within the offline muon reconstruction was tested, as demonstrated in Fig. 2 (right).
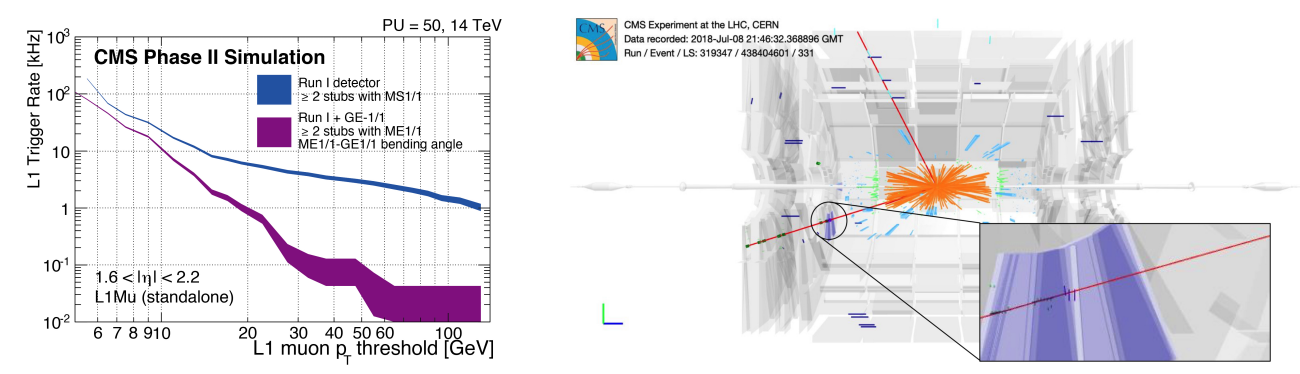

Figure 2: CMS L1 trigger rate in the region covered by GE1/1 detectors (left), computed with (purple) and without (blue) using information from GEMs. Event display showing a muon reconstructed using, among others, hits from GEM detectors (right).

\section{Performance of the muon system at HL-LHC}

The expected impact of the upgrade of the muon system to the CMS L1 muon trigger and to the offline reconstruction was extensively studied using simulations. The addition GEM and iRPC detectors in the muon endcaps increases the trigger efficiency, and the excellent spatial resolution of GEMs provides a more accurate standalone measurement of the $\mathrm{L} 1$ muon $p_{T}$, reducing trigger rates. An example is given in Fig. 3. The expected trigger efficiencies and rates are presented for $2.1<|\eta|<2.4$, a challenging region of the detector, which is characterized by high background rates and a low, nonuniform, magnetic field. In comparison with a CSC-only trigger, the results show that the use GEMs in the trigger allows for a rate reduction of approximately one order of magnitute and an increase of the efficiency of about $10 \%$. Moreover, the muon system upgrade will allow for the design of new triggers that will permit an efficient online selection of many Beyond the Standard Model (BSM) signatures. For example, the electronics upgrades of DT and RPC, as well as the addition of iRPCs, will allow us to measure the crossing time of a particle with a resolution of few ns within the trigger. This ability to measure the accurate timing of a particle opens the possibility of developing triggers to search for slow-moving particles as a signature for BSM.

Finally, the muon system upgrade will also improve the performance of the offline reconstruction. This is shown in Fig. 4, where muon identification efficiencies and background yields are studied using $Z \rightarrow \mu \mu$ and $t \bar{t}$ simulated samples. The acceptance is increased up to $|\eta|=2.8$, while maintaining reasonable control over the background multiplicity. In addition, when potential failures of the present muon system (estimated estrapolating consrevatively present failure rates to the 

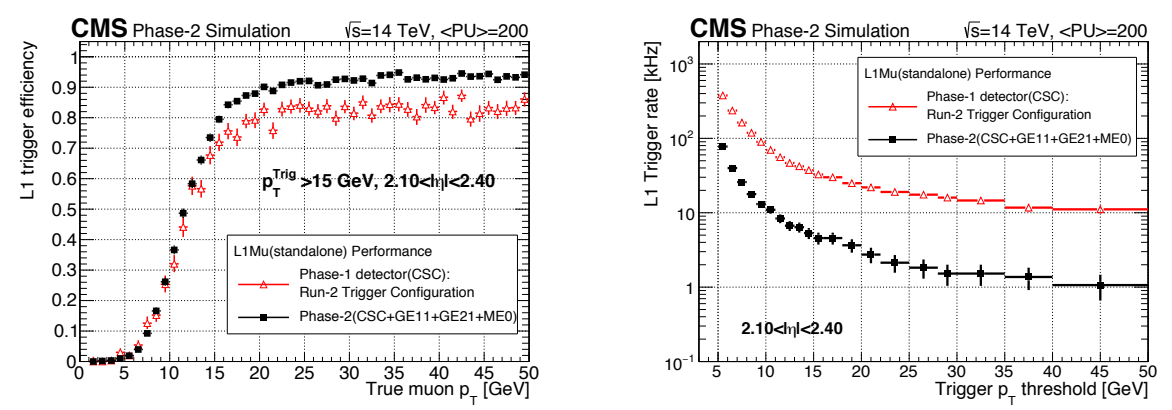

Figure 3: Trigger efficiency (left) and trigger rate (right) in the region covered by GE1/1, GE2/1 and ME0 detectors computed using (black) and not using (red) hits from GEMs.

end of HL-LHC) are considered, it is shown that the newly installed detectors help preserving high identification efficiency.
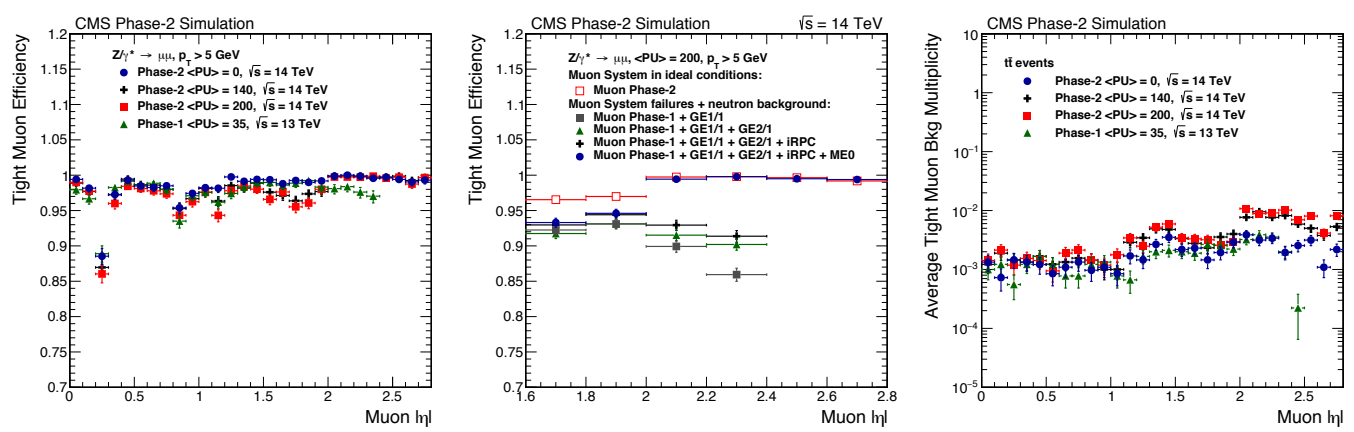

Figure 4: Tight offline muon identification efficiency (left, centre) and background yield (right) computed using muons from $Z \rightarrow \mu \mu$ and $t \bar{t}$ decays.

\section{Summary}

A comprehensive upgrade of the CMS muon system is planned to cope with the harsh condition of radiation levels and hit densities foreseen at HL-LHC. It features an upgrade of the electronics of existing detectors and the addition of new chambers in the forward region of the muon spectrometer. Simulation studies show that this upgrade will maintain or improve the excellent physics performance achieved until now by the CMS detector.

\section{References}

[1] G. Apollinari, et. al., "High-Luminosity Large Hadron Collider (HL-LHC) : Technical Design Report”, CERN Yellow Rep. Monogr. 4 (2017) 1. doi:10.23731/CYRM-2017-004

[2] The CMS Collaboration, "The Phase-2 Upgrade of the CMS Muon Detectors", CERN-LHCC-2017-012, CMS-TDR-016, 2017.

[3] The CMS Collaboration, "CMS Technical Design Report for the Muon Endcap GEM Upgrade", CERN-LHCC-2015-012, CMS-TDR-013, 2015. 